\title{
DETERMINATION THE AUTHENTICITY AND QUALITY OF THE GOODS
}

\author{
Sergey Minkov, Andrey Novikov ${ }^{1}$ \\ National Research Tomsk State University, 634050, Tomsk, Russia
}

\begin{abstract}
Often the question arises, how to assess the quality of the goods. To determine either fake or genuine product, there are many different methods and techniques, but there is no universal method for determining the low-quality products. It is proposed to provide a compact device for use with any mobile device, such as a cell phone, as well as the creation of algorithm and software that will allow the end user to easily use the device for self-determination of the authenticity and quality of the goods, comparing the results of the spectra analysis.
\end{abstract}

\section{Introduction}

Food safety is a long-standing problem for consumers. Even if the product was fresh during transportation to the place of sale or at a time when the buyer acquires it, the quality, freshness and safety at the time of use cannot be guaranteed. Thus, a method that will allow the buyer to check the food product in the presence of deviations (violations), immediately prior to use, it will be very convenient for the consumer.

\section{Problematic}

Non-invasive methods, i.e. methods that are not related to the direct use of the product in the study (testing), as well as possible better suited for use by consumers, as this method provides ease of use and therefore a higher probability of use. Spectroscopic methods for determining the quality of food products meet the requirements to be non-invasive and easy-to-use. But there are two major obstacles to be overcome before the use of such methods consumers.

The first is the need to establish an appropriate spectrometer. Figure 1 shows the structure of a typical spectrometer [1]. Light enters through the entrance slit, is reflected by the mirror 1 at a dispersive element such as a diffraction grating which directs light to a mirror 2. Since the grating disperses light according to its wavelength, each pixel of the CCD-spectrometer detector is lit a certain wavelength. Spectrometers that use this design are complex, require precise alignment, difficult to assemble, and are limited in their ability to miniaturization. The spectrometer is suitable for everyday use by the consumer should be small, durable, easy to use and have features that provide product testing sufficient accuracy and reliability [2].

\footnotetext{
${ }^{1}$ Corresponding author: unwell@yandex.ru
} 
The second obstacle is that foods - not simple matter but complex mixtures of substances, which themselves tend to have complex spectra. Thus, to be useful to the consumer, such a recognition system must include means for analyzing complex spectra obtained in real time.

When designing such a spectrometric analysis system should take into account that, for the consumer it would be useful to determine not only the quality and authenticity of the products, but also to apply the device in other areas. For example, such a system can be used in various applications such as the detection of environmental contaminants, detection of explosive and remote chemicals or biological agents, as well as remote diagnostics and monitoring a patient's condition (e.g., blood oxygen levels or glucose levels).

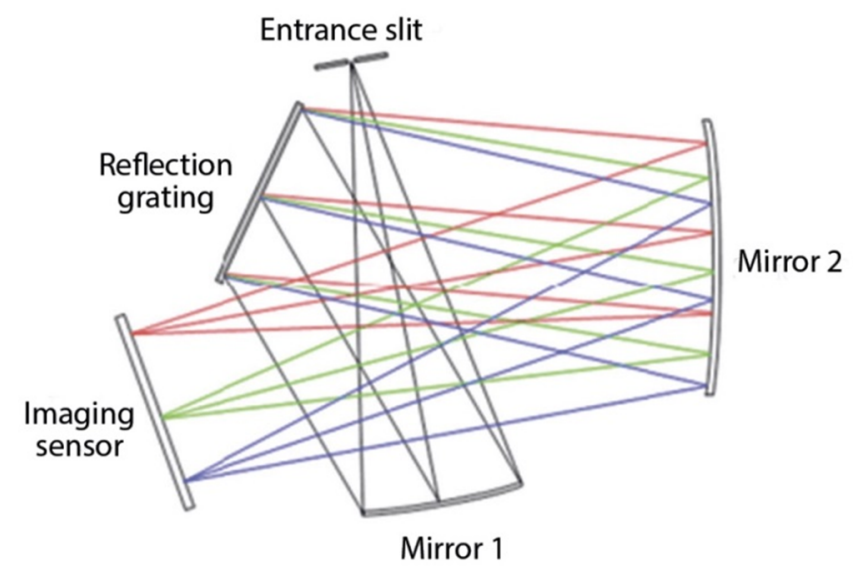

Figure 1. A typical spectrometer.

Compact spectrometer that can be integrated into consumer devices such as cellular phones must be sufficiently durable and have a low cost, to be convenient and practical for spectroscopic measurements performed by the user in a domestic environment. This is a convenient method by which to obtain the spectra (in particular from complex mixtures such as food) and analyze them to meet product quality and authenticity. The compact spectrometer system and its method of operation are designed to meet this need.

\section{Principle of operation}

The basic principle of a compact spectrometer is as follows (Figure 2). Light enters the optical filter comprising a plurality of sub-filters aligned along the same axis. These subfilters form a luminous flux on a plane-convex lens (a convex surface facing the optical filter). Several different signals are formed after the lens, which are dispersed along the micro-lens array onto the detector. A micro-lens array arranged so that light is scattered by the detector along an axis parallel to the axis which combines sub-filters.

The detector may be of any suitable type, able to detect light in the required range: from UV to IR radiation, depending on the application.

Using only single filter may restrict the available spectral range. For example, if the angle of incidence greater than $30^{\circ}$, the signal intensity is insufficient due to lens aberrations and this will reduce the efficiency of the detector at large angles [3]. For the angular range of $30^{\circ}$ and the optical dichroic filter more than $850 \mathrm{~nm}$ spectral range available for the spectrometer is $\sim 35 \mathrm{~nm}$. This range is sufficient for use in Raman spectroscopy [4-6]. To solve this problem, an optical filter is used, which consists of a plurality of auxiliary filters, wherein each sub-filter is a dichroic filter with a narrow width 
in its transmission spectrum wavelength range, and thus, can reach different parts of the optical spectrum. Depending on the number of sub-filters wavelength range available to the spectrometer, can reach hundreds of nm.

In the case of using a plurality of auxiliary filters plano-convex lens overlaps formed on the image plane (one from the sub-filter), and finally the signal received at any particular pixel of the detector is the result of a mixture of different signals collected through the lens. In order to separate the signals from different sub-filters, you must use an array of microlenses, which must be placed between the lens and the detector (the convex side to the detector). Such micro-lens arrays are well known and have been used, such as light-field camera [7].

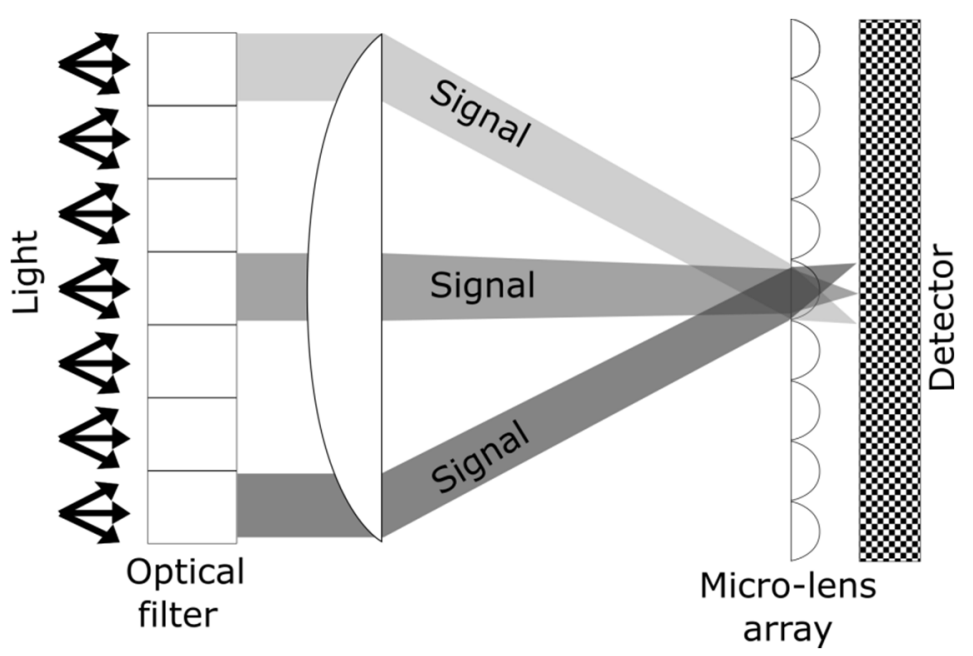

Figure 2. Compact spectrometer.

Their location coincides with the focal plane of the plano-convex lens and the detector plane. Each micro-lens acts as a "super-pixel", which separates the light incident on it from the lens, which in turn, received by a plurality of auxiliary filters. Each micro-lens generates an image at the detector aperture of the optical filter. Such a "micro-image" is a contribution to the signal of each portion (i.e., optical filters) lens aperture.

Sample measurement is performed through the use of the scattered ambient light. In most cases, the natural ambient light is not intense enough to provide a range of high enough quality. Thus, compact spectrometer system includes a light source. The laser or light emitting diode can be used as a light source.

Using the spectrometer is determined the absorption spectrum of the object. Preservation of the spectrum to a database and use it for comparison with newly acquired spectrum will allow to compare objects' characteristics with the characteristics of the reference object (in the database) to assess their authenticity and quality (Figure 3).

The image or sequence of images is captured by the image sensor in the spectrometer. The images are analyzed by a local processor. This step of analysis may include image averaging, compensation of the optical unit aberrations, detector noise reduction by using noise reduction algorithm or image transformation as the original spectrum.

Authenticity and quality are determined by the user independently by comparing the spectra results. For example, the user can read the coefficient spectra overlap greater than 0.8 as evidence that the compared spectra were obtained from identical objects.

The device operates only in conjunction with special software that implements spectral processing and matching algorithm. 
Today, mobile technologies cover more and more spheres of human activity. High availability of mobile devices, as well as benefits such as portability and low cost make the need to develop applications for mobile platforms. Therefore availability, ease of use and convenience of the software for the device is best implemented as applications for popular operating systems OS Android and iOS (Table 1).

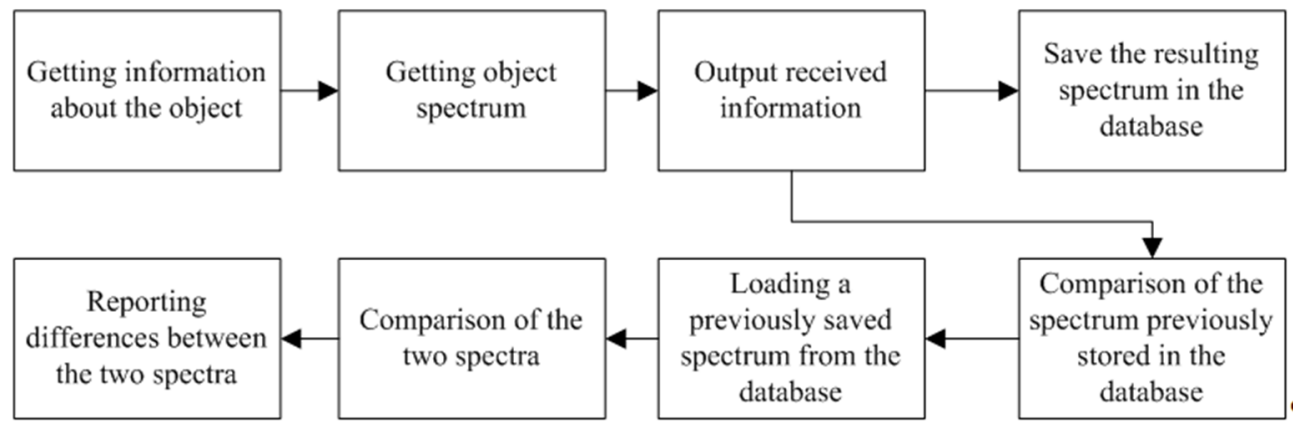

Figure 3. Information Model.

At the initial stage of the application is a native type using optimization design under multiple platforms with a single code base.

Table 1. Worldwide sales of smartphones to end users [8].

\begin{tabular}{|l|l|l|l|l|}
\hline \multicolumn{1}{|c|}{ OS } & \multicolumn{1}{|c|}{$\begin{array}{c}2015 \mathrm{Q} 2, \\
\text { thous. pcs. }\end{array}$} & $\begin{array}{c}\text { Market share in } \\
2015 \mathrm{Q} 2, \%\end{array}$ & $\begin{array}{c}\text { 2014 Q2, } \\
\text { thous. pcs. }\end{array}$ & $\begin{array}{c}\text { Market share in } \\
\text { 2014 Q2, \% }\end{array}$ \\
\hline Android & 271010 & 82.2 & 243484 & 83.8 \\
\hline iOS & 48086 & 14.6 & 35345 & 12.2 \\
\hline Windows Phone & 8198 & 2.5 & 8095 & 2.8 \\
\hline BlackBerry & 1153 & 0.3 & 2044 & 0.7 \\
\hline Other & 1229 & 0.4 & 1416.8 & 0.5 \\
\hline Total & 329676.4 & 100 & 290384.4 & 100 \\
\hline
\end{tabular}

\section{Conclusion}

As a result, an inexpensive reliable spectrometer without dispersion elements and moving parts will be created, as well as software for data analysis. Spectrometer system has no direct optical axis and in the selected embodiments includes no more than four elements. The required accuracy of alignment between the elements is very low, compared with a typical spectrometer. The absence of direct optical axis and the small size of the detector allows to organize the production of spectrometers, which are sufficiently small and economical to fit into devices such as cell phones, but in the other hand have sufficient sensitivity and resolution (typically $<10 \mathrm{~mm}$ ), to obtain spectra of complex mixtures, such as foods.

\section{References}

[1] K. Siegbahn, Alpha-Beta and Gamma-Ray Spectroscopy (North-Holland Publishing Co., Amsterdam, 1966)

[2] Opticheskie spectralnye pribory (The optical spectral instruments) / ed. I.V. Skokov (Mmashinostroenie, Moscow,1984) 
[3] D.W. Ward, K. A Nelson, K. J. Webb, New Journal of Physics 7, 213 (2005) doi: 10.1088/1367-2630/7/1/213

[4] Osnovy molekulyarnoi spektroskopii (Fundamentals of molecular spectroscopy)/ ed. Y.A. Pentin, G.M. Kuramshina) (M.: Mir, Moscow, 2008)

[5] Pietro Baraldi, Anna Tinti, MATEC Web of Conferences 36, 01002 (2015) doi: $10.1051 /$ matecconf $/ 20153601002$

[6] D. A. Skoog, F. J.Holler, S. R. Crouch, Principles of instrumental analysis (Thomson Brooks/Cole, Australia, 2007)

[7] E.H. Adelson, J.Y.A. Wang, IEEE Trans. on Pattern Analysis and Machine Intelligence 14, 2 (1992)

[8] IT-industry: the world economy against the IT. URL: http://pressenter.ru/index.pl ?act=PRODUCT\&id=2536 